\title{
Effect of social medical security on residents' health and economic burden of disease.
}

\author{
Bai Min* \\ School of Economics, Wuhan University of Technology, PR China
}

\begin{abstract}
Our objective is to explore and analyse the effect of social medical security on residents' health and economic burden of diseases. We analysed the national data of China to figure out the impact of medical security on the residents' health and the economic burden of disease. The investigation found that reasonable medical security played a key role in residents' health; with the improvement of medical security policies and the increase of government' investments, the residents paid much less for their diseases, reducing their economic burden. After generalizing the previous studies, it is not hard to find that a sound medical security improves the health of people and eases the economic burden of disease in positive, but some people don't agree with it for its lack of evidences.
\end{abstract}

Keywords: Social medical security, Residents' health, Disease economy.

Accepted on November 8, 2017

\section{Introduction}

In recent years, leaders of the party and nation attach importance to health-care which is a major issue of the nation's economy and the people's livelihood. The Ministry of Health of China has launched a strategic research named "Healthy China 2020" for citizens' health problems since 2008.

The national health resources should be used fully and effectively to meet the demand of masses for health, which promotes the residents' health [1]. With enough health resources before reform and open, the public health system has been built up step by step under the endeavour of the government. It is said that in 1980s, the Chinese mean life-span had increased by $68 \mathrm{y}$ from $35 \mathrm{y}$, the incidence of infectious diseases such as malaria had decreased by $0.3 \%$ from $5.5 \%$, and the mortality of new-born had reduced by $4 \%$ from $26 \%$, which evidenced that the health system of China made giant contribution to Chinese health [2]. To sum up the national health funds and the residents' economics, the cash outlay of the individual accounted for $20.50 \%$ of the total and the payment of the central finance department the majority in 1978. Since the reform and open, our public health system has got corresponding changes, and the corresponding health standards established quicken the legality development of public health. In recent years, the government has increased the total expenditure for health and has improved some health issues. However, given all kinds of medical issues and status, the expenditure is inadequate [3], and various managements involving health should be perfected. Consequently, we conduct a study about the government health expenditure and its effect on the residents' health, and propose some ideas to the polices based on the findings.

\section{Review}

\section{Relationship between social medical security and residents' health}

Through comparing and analyzing the heath of the residents with or without social medical security, the study of social medical security and economic burden of disease was conducted. The related factors included population control, medical techniques improvement, and health. An empirical study about the health of the residents with or without medical security was implemented with controlling the factors such as population control, medical techniques, and health behaviors. Compared the health condition of the adults aged from 50 to 60 $y$, it was known that the citizens with insurance were more health than those without it, suggesting medical security safeguarded the health of the middle age and the elderly people [4]. Literature research revealed the effect of medical security on the mortality and the relationship between medical security and the two weeks incidence of the residents. And it was also found that the participation rate of medical insurance for urban residents was negatively related to the two weeks incidence of the residents. Especially, the medical security could improve the health of the residents over $65 \mathrm{y}$. In terms of the study on the relationship between medical insurance and the residents' self-assessment about their health condition, the data of "Chinese Health and Nutrient Survey (CHNS)" in a related literature demonstrated that the new rural cooperative medical care system played a positive role in the farmers' health, but their health improvement was limited, and this system should be furthered in future medical development [5]. The studies mentioned above indicated that medical security had positive 
effect on the residents' health. However, some other studies believed that medical security had no significant impact on the residents' health. Some study found that the national medical security variously influenced the whole residents, and the medical security was mainly used to reduce the expenditure on health, raise the quality of medical service for residents, and promote the development of social preventive health care.

\section{Effect of medical security on economic burden of disease}

A review revealed that the relationship between medical security and economic burden of disease, and through compared the economic burden of the residents with or without health insurance, scholars suggested that the useful health insurance lessened the burden apparently [5-7]. The main insurances residents took out involve urban employee medical insurance, socialized medicine, and commercial health insurance, which greatly decrease the expenditure for health of the residents and disease economic burden, to reduce the total economic burden obviously. Medical security is essential for the resident aged from 50 to $60 \mathrm{y}$. Commonly, chronic diseases like hypertension burden the economy, and the health insurance compensation policy for hospitalization contributes to subside the financial pressure of the in-patients. A study discovered that the medical security shared a lot of risks of aliments, largely relieving the pressure for the low income family $[8,9]$. Some studies also showed that the aged with medical security might cost much more for health care, and they went to see a doctor easier but had a heavier economic burden. Why? the reason was that they got "money illusion", namely their demands for health care were increased by medical security, raising their medical expenditure rather than reducing. In a word, the overseas and domestic scholars conducted many investigations about the interrelationship of medical security, residents' health, and disease economic burden as well, but there were some debates. Some scholars considered that the causal relationship of them didn't convince everyone, and the findings of some studies revealed that there were many influencing factors between health insurance and the residents' health. Yet some scholars confirmed the relationship between them through researches and supported that health insurance conduced to improve the residents' health and reduce disease economic burden.

\section{Macro data analysis on medical security and residents' health and disease economic burden improvement}

Through the study about the data of the third national health investigation, we found that the proportion of catastrophic health expenditure in China in 2003, 2008, and 2013 was $12.2 \%, 14 \%$, and $12.9 \%$ respectively [10]. And the expenditure had no change as against the orderly growth of the people's living standard. Nowadays, the basic medical insurance system for residents in China has been refined in constant, and the medical compensation has been raised ceaselessly, and medical insurance aids for the serious illnesses and a expenses claims system for 20 severe diseases has been launched, which relieves the problem that it is difficult and expensive for the common people to see doctors and solve their health and economic issues at maximum. In addition, the government also sets up chronic diseases insurance to help those who need to take medicine for a long time. However, it is proved that the medical compensation policies hereinbefore only reduce the family medical payments to a certain extent and has limited impact on the catastrophic health issues. Therefore, about the relationship between medical security and the residents' health as well as disease economic burden, it is known that health insurance isn't a major measure to reduce economic burden so that the government has to increase the proportion of medical compensation in proper from many aspects, but also control the medical payments rise from the medical supply departments, so as to protect the health of people and reduce resources waste at the same time.

\section{Discussion}

After generalizing the previous studies, it is not hard to find that a sound medical security improves the health of people and eases the economic burden of disease in positive, but some people don't agree with it for its lack of evidences. With the macro national data analysis, it is known that the continues improvement of citizen health is associated with the national economic and social development, living standard improving, advance in the medical techniques, and medical security systems refining as well [7]; the total medical expenditure in China has raised rapidly, but the individual payment reduces with the constant development of medical security system since 2001, besides, the people's health is improved with longer mean lifetime. To sum up, the social medical security guarantees the life and health of citizens, and reduces their economic burden of disease, yet with the advance of society, the health insurance system should keep its pace of improvement to meet the increasing demand for heath.

\section{References}

1. Chen Y, Ginger ZJ. Dose health insurance coverage lead to better health and educational outcome? Evidence from rural China. J Health Econ 2012; 31: 1-14.

2. Haung F, Wu CJ. Old age mortality crossover in China: does the public health insurance matter. Popul Res 2010; 34: 95-105.

3. Baker DW, Sudano JJ, Albert JM. Lack of health insurance and decline in overall health in late middle age. $\mathrm{N}$ Engl J Med 2001; 345: 1106-1112.

4. Zhou H. Study on the effect of basic medical insurance for urban residents on their health. Xiamen: Xiamen Univ 2014.

5. Wu LC, Shen SG. A empirical study on the effect of new rural cooperative medical system on the health of farmers. Insur Studies 2010; 12: 60-68.

6. Kowalski AE. What do longitudinal data on millions of hospital visits tell us about the value of public health 
insurance as a safety net for the young and privately insured. Soc Sci Electr Publ 2015; 13: 10-13.

7. Huo L, Chen Y. The new rural cooperative medical insurance system: do farmers get happiness. J Shanghai Univ Finance Econ 2017; 14: 21-24.

8. Hu HW, Liu GE. Impact of urban resident basic medical insurance on national health: effect evaluation and evidence of mechanism. South Chin J Econ 2012; 10: 186-199.

9. Zhou Z, Zhu L, Zhou Z. The effects of Chinas urban basic medical insurance schemes on the equity of health service utilisation: evidence from Shaanxi Province. Int $\mathrm{J}$ Equ Health 2014; 13: 23.
10. Li XJ, Wang ZH, Ling ZP. Analysis on the effect of new rural cooperative medical system on seeing doctors and health of farmers. World Econ Paper 2012; 3: 58-75.

\section{*Correspondence to}

Bai Min

School of Economics

Wuhan University of Technology

PR China 